\title{
Thinning of the Motor-Cingulate-Insular Cortices in Siblings Concordant for Tourette Syndrome
}

\author{
Cherine Fahim · Uicheul Yoon · Paul Sandor • \\ Kirk Frey · Alan C. Evans
}

Received: 17 February 2009/ Accepted: 13 July 2009/Published online: 9 September 2009

(C) Springer Science+Business Media, LLC 2009

\begin{abstract}
Fraternal twin studies on normal subjects have demonstrated low heritability (intra-class correlation coefficient) estimates for frontal brain regions $(r=0.43)$. Here we aimed to investigate the relatedness/similarity estimates of the frontal brain regions in fraternal subjects concordant for Tourette syndrome (TS). We sought to identify regional brain similarities between siblings concordant for TS as an exploratory step towards the identification of potential brain structures involved in the TS phenotype. The identified brain structures may then serve in subsequent molecular genetic and linkage studies. In addition, we regressed cortical thickness and TS clinical severity scores to assess the relation between TS clinical symptoms and cortical structures. Sixteen sibling pairs concordant for TS were scanned using a $1.5 \mathrm{~T}$ magnetic resonance imaging scanner (age range 10-25, mean $17.19 \pm 4.1)$. Brain morphology was assessed using the
\end{abstract}

C. Fahim $(\bowtie) \cdot$ U. Yoon · A. C. Evans

Department of Neurology and Neurosurgery, McGill University, Montreal, Canada

e-mail: cherine.fahimfahmy@mcgill.ca; cherine.fahim@gmail.com

A. C. Evans

e-mail: alan.evans@mcgill.ca

C. Fahim

Laboratory for the Experimental Research in Behavior (LERB),

Faculty of SSP, Institute of Psychology, University of Lausanne,

Lausanne, Switzerland

C. Fahim

Department of Psychiatry, University of Montreal, Montreal, Canada

C. Fahim $\cdot$ U. Yoon $\cdot$ A. C. Evans

McConnell Brain Imaging Center, Montreal Neurological Institute, McGill University, 3801 University Street, Montreal, QC H3A 2B4, Canada fully automated Civet pipeline at the Montreal Neurological Institute. TS was assessed using the Children's YaleBrown Obsessive Compulsive Scale (CY-BOCS), Yale Global Tic Severity Scale (YGTSS) and the Goetz Tic Scale. We report high relatedness/similarity estimates for fraternal siblings concordant for TS $(r=0.86-0.60)$ in the middle frontal-motor/cingulate/insular cortices. Regression analysis revealed significant negative correlations in the right insula with the YGTSS $(r=-0.41, F=6.09$, $P<0.02)$ and the left cingulated cortex with the (CYBOCS) $(r=-0.35, F=4.30, P<0.05)$. Since previous findings have concluded that normal fraternal siblings are less alike in frontal cortices, the present findings may be attributed to TS. We speculate that the high ICC between siblings and the negative correlation between TS symptoms severity and cortical thickness measurements are related to the disturbances in the maturation of the motor-cingulate-

\section{Fahim}

Sainte-Justine Hospital Research Centre, Montreal, Canada

\section{A. C. Evans}

Department of Biomedical Engineering, McGill University, Montreal, Canada

P. Sandor

Division of Brain Imaging \& Behaviour Systems-Neuroscience

Toronto Western Research Institute, University of Toronto,

Toronto, Canada

K. Frey

Departments of Radiology and of Neurology, Mental Health Research Institute, University of Michigan, Ann Arbor, USA 
insular cortical neural system that mediate self-regulatory processes. Such delayed maturation may consequently contribute to the development of TS by releasing motor and vocal tics from regulatory control. These findings may have important genetic implications.

Keywords Tourette syndrome - Cortical thickness . Neuroimaging - Anterior cingulate $\cdot$ Insula $\cdot$ Motor · Similarity $\cdot$ Intra class correlations

\section{Introduction}

Tourette syndrome (TS) present with a substantial genetic contribution, with no clearly identified genes (Pauls 2003; Robertson and Cavanna 2007; Tourette Syndrome Association International Consortium for Genetics 2007; Verkerk et al. 2006; Laurin et al. 2009). Hence, emerges the need for discovering neuroendophenotypes that may increase the power to detect quantitative traits influencing behavior and disease liability. Giedd et al. (2007) stated that highly heritable brain morphometric measures provide biological markers for inherited phenotypes, and may serve as potential targets for genetic linkage and association studies. Therefore, we sought to identify regional brain similarities in siblings concordant for TS as an exploratory step towards the identification of potential brain structures involved in the TS phenotype. The identified brain structures may then serve in subsequent molecular genetic and linkage studies. TS is a neurodevelopmental disorder involving the disinhibition of the cortico-striatal-thalamiccortical circuitry (CSTC) (Leckman et al. 1998). In 1991, Leckman and colleagues argued that facial tics would be associated with dysfunction in an orofacial subset of the motor circuit, eye blinking with the occulomotor circuit, whereas lack of inhibition or response selection would be linked to a dysfunction in the prefrontal cortex.

A study by Thompson et al. (2001) concluded that the sensorimotor and parietal occipital but not frontal territory is significantly more correlated in fraternal twins. Yet, monozygotics (MZ) are almost perfectly correlated in their gray matter (GM) distribution, with near-identity in the frontal GM. Similarly, Wallace and colleagues (2006) found the frontal GM volume intraclass coefficient correlation (ICC) to be 0.46 in dizygotic twins (DZ) versus 0.82 in MZ. Our group found frontal ICC and estimates of heritability to be 0.43 in DZ versus 0.71 in MZ 8-year-old twins (Yoon et al. 2008). In addition, using the fully automated Civet pipeline at the Montreal Neurological Institute, Peper et al. (2009) found high heritability in $107 \mathrm{MZ}$ and DZ twin pairs in the posterior fronto-occipital, corpus callosum, and superior longitudinal fascicles (up to 93\%), and the amygdala, superior frontal and middle temporal cortices (up to 83\%). Using the same methodology, Lenroot et al. (2009) found regions within the dorsal frontal and temporal cortices to be significantly heritable, consistent with previous the previous studies. The authors additionally found areas of significant heritability in the orbitofrontal cortex, superior parietal regions, and inferior surfaces of the temporal lobes. Overall these studies investigated heritability estimates in normal subjects. However, to the best of our knowledge no prior study investigated brain heritability estimates in siblings concordant for TS. Based on the above, we used ICC to measure frontal cortical thickness in 16 fraternal siblings concordant for TS. Such an approach would allow us to determine frontal cerebral cortex relatedness/similarity estimates. A priori we predicted that frontal cortices between siblings concordant for TS would show significant ICC similarities based on the TS behavioral phenotype. In this vein, we regressed cortical thickness and TS severity scores to assess how TS affects cortical morphology. The ICC is used to estimate the similarity of one variable between two members within a group. The ICC ranges from 1.0 to -1.0 . It is large and positive when there is little variation within the pairs but the means between the pairs differ. It is large and negative when the variation within a pair is much greater than that between the pairs. Cortical thickness is of particular interest in assessing the ICC estimates between siblings concordant for TS. TS results in the disinhibition of the cortex (Leckman et al. 2001). Cortical folding affects cellular and dendritic shape, as well as the layout of cortical blood vessels (Miodonski 1974). These effects are most evident in prefrontal cortices (Hilgetag and Barbas 2005). Genetic factors play an important role in the timing of cortical neuronal migration during development (Rakic 1995) and determine the density and layout of connections, as the cortical sheet expands and folds.

\section{Methods}

Subjects

Thirty-four subjects were recruited from families who participated in the TS Genetic Linkage Study: 16 sibling pairs concordant for rigorously diagnosed and characterized TS. Subjects were predominantly right-handed (90\%) according to the Edinburgh inventory (Oldfield 1971). TS was assessed using the DSM-IV (American Psyciatric Association 1994), the Children's Yale-Brown Obsessive Compulsive Scale (CY-BOCS) (Scahill et al. 1997), the Yale Global Tic Severity Scale (YGTSS) (Leckman et al. 1989) and the Goetz Tic Scale (Rush Scale) (Goetz et al. 1987) (Tables 1 and 2). 
Table 1 Subjects characteristics

\begin{tabular}{ll}
\hline Characteristics & Tourette syndrome \\
\hline Age in years & $17.19 \pm 4.1$ \\
& $(\mathrm{~B}=16.87 \pm 4.17 ; \mathrm{G}=17.95 \pm 4.00)$ \\
CY-BOCS & $(\mathrm{M}=1.24 \pm 1.21 ; \mathrm{F}=1.07 \pm 1.16)$ \\
Gender & $32(\mathrm{M}=22 ; \mathrm{F}=10)$ \\
Rush scale & $(\mathrm{M}=5.29 \pm 2.53 ; \mathrm{F}=5.21 \pm 2.72)$ \\
YGTSS & $(\mathrm{M}=28.03 \pm 25.07 ; \mathrm{F}=28.31 \pm 23.74)$
\end{tabular}

$F$ Females, $M$ Males, $C Y$-BOCS Children's Yale-Brown Obsessive Compulsive Scale, YGTSS Yale Global Tic Severity Scale, Rush Tic Rating Scale Goetz Tic Scale
Morphological Magnetic Resonance Imaging (MRI) Acquisition

Siemens Magnetom SonataVision syngo 1.5T Montreal Neurological Institute (MNI). T1 mprage sequence (scan time $8 \mathrm{~min}, 6 \mathrm{~s}$; pixel $0.98 \times 0.98 \mathrm{~mm})$. TR $=9.7 \mathrm{~ms}$; $\mathrm{TE}=4 \mathrm{~ms} ; \mathrm{TI}=300 \mathrm{~ms} ; \mathrm{TD}=0 \mathrm{~ms}$ using a $12^{\circ}$ flip angle. Number of slabs $=1$ fixed; slab thickness $=160 \mathrm{~mm}$; slice thickness $=0.98 \mathrm{~mm}$; number of partitions $=164 ; 3 \mathrm{D}-\mathrm{OS}=0 \%$; matrix $266 * 256$.

Table 2 Mean and standard deviation of the motor-cingulate-insular cortices in each of the 16 sibling pairs

\begin{tabular}{|c|c|c|c|c|c|c|c|c|c|c|}
\hline Family & Age & Gender & $\begin{array}{l}\text { Right middle } \\
\text { frontal-motor } \\
\text { (\# of vertices } \\
=416 \text { ) }\end{array}$ & SD & $\begin{array}{l}\text { Right insula } \\
\text { (\# of vertices } \\
=75 \text { ) }\end{array}$ & SD & $\begin{array}{l}\text { Left cingulate } \\
\text { (\# of vertices } \\
=602 \text { ) }\end{array}$ & SD & $\begin{array}{l}\text { Left middle } \\
\text { frontal-motor } \\
\text { (\# of vertices } \\
=25 \text { ) }\end{array}$ & SD \\
\hline 1 & 21.38 & M & 3.6906 & 0.0682 & 5.0519 & 0.2807 & 3.3885 & 0.1351 & 3.8944 & 0.0524 \\
\hline 1 & 24.47 & $\mathrm{~F}$ & 3.806 & 0.0818 & 4.9563 & 0.2397 & 3.3917 & 0.1857 & 3.9899 & 0.0129 \\
\hline 2 & 15.67 & M & 3.7497 & 0.0617 & 5.1842 & 0.1699 & 3.6585 & 0.1192 & 3.8737 & 0.0156 \\
\hline 2 & 15.67 & M & 3.7101 & 0.0887 & 5.0126 & 0.195 & 3.624 & 0.1488 & 3.902 & 0.0269 \\
\hline 3 & 13.77 & M & 4.4716 & 0.165 & 4.9944 & 0.2234 & 3.782 & 0.1328 & 4.3573 & 0.0477 \\
\hline 3 & 11.48 & M & 4.5718 & 0.1478 & 5.0745 & 0.2261 & 3.8459 & 0.1551 & 4.5721 & 0.0321 \\
\hline 4 & 15.73 & $\mathrm{~F}$ & 3.9448 & 0.1452 & 4.6261 & 0.1699 & 3.5972 & 0.1474 & 4.0127 & 0.0252 \\
\hline 4 & 19.07 & M & 3.8978 & 0.1255 & 4.4808 & 0.1576 & 3.5379 & 0.124 & 4.009 & 0.0427 \\
\hline 5 & 15.72 & M & 4.2667 & 0.2698 & 4.8639 & 0.227 & 3.7403 & 0.1006 & 4.5084 & 0.0253 \\
\hline 5 & 13.79 & $\mathrm{~F}$ & 4.1855 & 0.1856 & 4.8638 & 0.2321 & 3.7364 & 0.0938 & 4.4208 & 0.0158 \\
\hline 6 & 14.78 & M & 3.8764 & 0.2521 & 4.5688 & 0.2298 & 3.7899 & 0.0919 & 4.2587 & 0.0333 \\
\hline 6 & 13.17 & M & 4.0813 & 0.1705 & 5.0468 & 0.2372 & 3.7311 & 0.1427 & 4.4256 & 0.0198 \\
\hline 7 & 23.01 & M & 4.0842 & 0.1852 & 5.2713 & 0.1555 & 3.5328 & 0.1682 & 4.1888 & 0.0371 \\
\hline 7 & 18.24 & M & 4.1591 & 0.1682 & 4.9791 & 0.2055 & 3.5918 & 0.2085 & 4.3568 & 0.0322 \\
\hline 8 & 11.47 & M & 4.1872 & 0.1529 & 4.9336 & 0.117 & 4.0182 & 0.113 & 4.751 & 0.0377 \\
\hline 8 & 9.49 & M & 4.1611 & 0.0852 & 5.1098 & 0.1782 & 4.0426 & 0.1338 & 4.5436 & 0.0438 \\
\hline 9 & 16.87 & M & 3.8003 & 0.0927 & 4.3955 & 0.1287 & 3.49 & 0.0745 & 3.9238 & 0.0585 \\
\hline 9 & 19.51 & M & 3.9202 & 0.1134 & 4.3594 & 0.1371 & 3.4609 & 0.1368 & 3.9976 & 0.0217 \\
\hline 10 & 18.74 & M & 4.1526 & 0.1888 & 4.7189 & 0.2565 & 3.6689 & 0.1387 & 4.1264 & 0.0201 \\
\hline 10 & 17.38 & F & 4.0156 & 0.1661 & 4.8501 & 0.1929 & 3.4976 & 0.1219 & 4.6131 & 0.0322 \\
\hline 11 & 16.46 & M & 3.8916 & 0.2006 & 4.5977 & 0.2 & 3.5369 & 0.0938 & 4.0178 & 0.022 \\
\hline 11 & 19.77 & M & 3.7509 & 0.1323 & 4.3432 & 0.1254 & 3.5646 & 0.1315 & 3.9717 & 0.0107 \\
\hline 12 & 17.04 & $\mathrm{~F}$ & 3.7468 & 0.1849 & 4.1206 & 0.0936 & 3.716 & 0.1405 & 4.3 & 0.0153 \\
\hline 12 & 13.81 & $\mathrm{~F}$ & 3.8764 & 0.0827 & 4.4772 & 0.1861 & 3.6432 & 0.1693 & 4.0844 & 0.016 \\
\hline 13 & 14.5 & $\mathrm{~F}$ & 4.0528 & 0.0969 & 4.6128 & 0.1693 & 3.6244 & 0.1775 & 4.3051 & 0.026 \\
\hline 13 & 17.16 & $\mathrm{~F}$ & 4.0776 & 0.1929 & 4.9297 & 0.2177 & 3.6626 & 0.1415 & 4.3836 & 0.0205 \\
\hline 14 & 25.9 & M & 3.8217 & 0.1923 & 4.7743 & 0.1948 & 3.4567 & 0.0891 & 3.8211 & 0.0308 \\
\hline 14 & 23.59 & F & 3.7122 & 0.1511 & 4.6567 & 0.2037 & 3.2719 & 0.0895 & 3.8449 & 0.0041 \\
\hline 15 & 20.83 & M & 4.0437 & 0.1435 & 4.7792 & 0.1907 & 3.4548 & 0.1914 & 4.2593 & 0.0278 \\
\hline 15 & 23.19 & M & 3.9786 & 0.0682 & 5.094 & 0.2184 & 3.3797 & 0.1408 & 4.1615 & 0.0145 \\
\hline 16 & 13.44 & M & 4.107 & 0.1567 & 4.5806 & 0.1281 & 3.5825 & 0.0991 & 4.1506 & 0.0112 \\
\hline 16 & 15.17 & M & 4.1156 & 0.1629 & 4.5826 & 0.1329 & 3.6808 & 0.1507 & 4.029 & 0.0194 \\
\hline
\end{tabular}

Note: The significant number of vertices are depicted between brackets beside each regions' name. Please note that outstanding similarity between each sibling pair in the cortical thickness of motor-cingulate-insular regions 


\section{MRI Data Analysis}

Cortical thickness analysis was done using the fully automated CIVET pipeline (Lyttelton et al. 2007; Robbins et al. 2004; Zijdenbos et al. 2002; Sled et al. 1998). (1) Quality Control of the T1-weighted images: The CIVET automated quality control (QC) mechanism relies on measuring how far each point in a brain image lies from its reference distribution. This reference distribution is derived from the group of scans, which are submitted in the CIVET run. Armed with the mean and standard deviation images for the reference distribution, a "deviance" image can be produced for any subject scan which measures the distance of each value in the subject's image from the mean of the reference distribution, in standard deviations. Accordingly, for a value of " 2.5 " at a location in the image, it would be interpreted that "The value of the subject image at this point lies 2.5 reference standard deviations away from the reference mean." The outlier images can then be processed to produce a single statistic that highlights the particular type of outlier that is being detected. This general measure can be interpreted as an "outlier image". (2) Pre-processing the native files: These stages create links to the source image files within the 'native' subdirectory of the output directory. Later stages will operate on these links. (3) The 'non-uniformity correction' stages: An artefact often seen in MRI is for the signal intensity to vary smoothly across an image. Variously referred to as radio frequency (RF) inhomogeneity, shading artefact, or intensity non-uniformity, it is usually attributed to such factors as poor RF field uniformity, eddy currents driven by the switching of field gradients, and patient anatomy both inside and outside the field of view. Using N3, these stages will run an initial correction of intensity non-uniformity in the native images. (4) The 'registration' stages: Registration is the process of the alignment of medical image data. In brain-imaging studies, there is a need to put all image volumes into the same spatial coordinate system (stereotaxic space), by aligning all the images to a pre-defined atlas or template brain. This provides a way to compare data from similar locations in different brains and allows quantitative analysis. By default, CIVET uses the template generated from 152 subjects in the ICBM project, which is the template often used to bring images into what is referred to as MNI-Talairach stereotaxic space. (5) The 'final' stages: Since the transformations necessary to bring source images into MNI-Talairach space had been computed in the previous stages, now we need to 'resample' the images, essentially applying the computed transformation on the actual images. This means that each voxel will acquire a new position in space given by the spatial dimensions specified by the computed transformation. The output is the 'final' image, or the image in stereotaxic space. (6) Second non-uniformity corrections: Since we have noticed qualitative improvements in subsequent steps when an additional run of N3 (correction of intensity nonuniformity) is performed in stereotaxic space, these stages do just that. This is the default set-up. The user may elect to run non-uniformity corrections only once (either prior to or after the linear registration stages). (7) The 'classification' stages: These are the steps that produce 'discretely' classified (segmented) images from the final images. Basically, the intensity of each voxel puts it into one of 4 categories: GM, white matter (WM), CSF, or background. To do this, INSECT uses a set of standard sample points (or tags) in the brain volume, which have high probability of belonging to assigned tissue types. These tags are then used to compute an 'initial' volume classification. This classification is then used to purge incorrect tag points from the standard set, thus yielding a custom set of labels for the particular subject. The tag point set is then used by an artificial neural network (ANN) classifier to classify the volume. (8) The 'masking' stages: This step eliminates the skull and meninges by creating a course cortical surface then removing tissue lying outside of it. The surface produced here is a result of a deforming ellipsoid polygonal model that shrinks inward in an iterative fashion until it finds the pial surface of the cortex. Because it does not converge completely towards the pial surface of the cortex, it is not accurate enough to become the final pial surface in our corticometric analysis. But it does a decent job as a mask to exclude the skull and meninges. This masking component of the pipeline is undergoing development currently and will likely be improved a great deal soon. (9) The 'partial volume estimation' stages: These stages will calculate the partial volumes in each voxel. This essentially means that the volume will be classified 'continuously' instead of 'discretely'. Therefore, for each voxel, there will be a percentage of GM, WM, CSF, and background, depending both on the voxel's intensity and on the neighbouring voxels' intensities. This is especially useful information when examining voxels that lie on, or cross the boundary between tissue types (e.g. voxels around the pial surface of the cortex), and in deep narrow sulci. This will be important for later stages that attempt to accurately produce the cortical surfaces. In addition, a 'skeletonised CSF' will be produced: A continuous layer of CSF surrounding the pial surface of the cortex. This will also be a requisite to produce cortical surfaces. (10) The volumetric nonlinear-fitting' stage: Non-linear registration is the set of techniques that allow the alignment of data sets that are mismatched in a non-linear or non-uniform manner. This involves deforming or warping the input brain-image until it becomes nearly identical to the registration target. A "deformation field" is thus produced, which essential contains the all the information necessary to transform each 
voxel of the input image into the corresponding voxel of the target image. Non-linear registration to the registration target (in this case a probabilistic atlas) will allow us to use ANIMAL. So this stage calculates the transform necessary for this kind of registration. (11) The 'ANIMAL' stages: ANIMAL essentially maps the images to a probabilistic atlas developed from the ICBM database. Brain lobes and major brain regions and structures are identified in the atlas, and each voxel is then given a probability value of being in that lobe or structure. These stages will also calculate the volume of the identified lobes. (12) The 'smoothmatter' stages: The smooth-matter stages basically run a smoothing (blurring) kernel on the different tissue classes of the brain. These steps are prerequisites for the purposes of examining symmetry in subsequent stages. (13) The 'symmetry analysis' stages: These steps produce output that allows for the analysis of symmetry/asymmetry of brain tissues. (14) The 'cortical fitting' stages: The surfaces produced here by CLASP are a result of a deforming ellipsoid polygonal model that shrinks inward in an iterative fashion until it finds the inner surface of the cortex that is produced by the interface between GM and white matter. This surface is often referred to as the 'white-surface'. The surface is a polygonal (triangulated) mesh, each point on which is referred to as a 'vertex'. Once this surface is produced, a process of expansion outwards towards the CSF skeleton follows. This process is governed by laplacian fluid dynamics and attempts to find the best fit for the pial surface (or grey-surface) taking into account the partial volume information. Since this surface is an expansion from the white-surface, each vertex on the new surface is 'linked' to an original vertex on the white-surface. Optionally, a polygonal mesh with $327 \mathrm{~K}$ triangles (instead of the default $81 \mathrm{~K}$ mesh) could be produced, thereby quadrupling the number of vertices. (15) The ' $t$ _link' stages: Since each vertex on the grey-surface is linked to vertex on the white-surface, a reliable metric to measure cortical thickness is the distance between linked vertices. This is more likely to be biologically meaningful than many other metrics of cortical thickness, and is referred to as the 't_link' metric. These stages calculate the t_link thickness in stereotaxic space, then in native space. The latter is achieved by applying the reverse of the linear transform on the volume (therefore taking the volume back to native space), then calculating thickness. Both sets of cortical thickness values are then smoothed using a diffusion-smoothing kernel that is applied on the cortical surface. (16) The 'cortical parcellation' and 'surface area' stages: Once the surfaces, thickness values and the ANIMAL labels have been produced, it is now possible to intersect the labels of the brain lobes with the cortical surfaces. This will allow the calculation of mean cortical thickness values for these lobes, as well as an estimate of cortical surface area for each lobe. All of this is done in native space. (17) The 'non-linear surface registration' stages: Once the cortical surfaces are produced, they need to be aligned with the surfaces of other brains in the data set so cortical thickness data could be compared across subjects. To achieve this, SURFREG performs a non-linear registration of the surfaces to a pre-defined template surface. This transform is then applied (by resampling) in native space. Note that while the vertices have been aligned, the topological measurements associated with them (e.g. thickness), remain unchanged in this process. (18) The 'verification' stage: For purposes of rapid quality assessments of the output of this pipeline, these stages produce an image file in '.png' format that show-cases the output of the main stages of the pipeline. A 30-mmbandwidth blurring kernel was applied; this size was chosen on the basis of population simulations which that this bandwidth maximized statistical power while minimizing false positives (Lerch and Evans 2005). This kernel allows anatomical localization, as $30 \mathrm{~mm}$ blurring along the surface using a diffusion smoothing operator preserves cortical topologic features and represents considerably less cortex than the equivalent volumetric Gaussian blurring kernel. The threshold for statistical significance was set at an $\alpha$ of 0.05 . Correction for multiple comparisons was needed to control the false-positive rate. The false discovery rate (FDR) controlling procedure for multiple comparisons is reported to be effective for the analysis of neuroimaging data. Of particular note, In 2003, our group (Chung et al. 2003) presented a unified statistical approach to deformation-based morphometry applied to the cortical surface specifically when using age and gender as covariates. The cerebral cortex has the topology of a 2D highly convoluted sheet. As the brain develops over time, the cortical surface area, thickness, curvature, and total GM volume change. It is highly likely that such age-related surface changes are not uniform. By measuring how such surface metrics change over time, the regions of the most rapid structural changes can be localized. We avoided using surface flattening, which distorts the inherent geometry of the cortex in our analysis and it is only used in visualization. To increase the signal to noise ratio (SNR), diffusion smoothing, which generalizes Gaussian kernel smoothing to an arbitrary curved cortical surface, has been developed and applied to surface data (2D smoothing). As an illustration, our group has demonstrated how this new surface-based morphometry can be applied in localizing the cortical regions of the gray matter tissue growth and loss in the brain images longitudinally collected in the group of children and adolescents. Lerch and Evans (2005) stated that each of the segmentation, thickness computation, and surface registration procedures are expected to introduce noise in the thickness measure. To counteract 
this, data smoothing was used to increase the SNR and the sensitivity of statistical analysis. For analyzing data in 3D whole brain images Gaussian kernel smoothing is widely used, which weights neighbouring observations according to their 3D Euclidean distance. In the present study, however, the data lie on a 2D surface so the smoothing must be weighted according to distance along the surface. This method is adopted to reduce the noise in the thickness measure especially when covarying with age and gender. Diffusion smoothing, that smooths data on an explicit 2D cortical surface representation, is based on the observation that, in Euclidean space, Gaussian kernel smoothing is equivalent to solving an isotropic diffusion equation. This diffusion equation can also be used on the surface manifold to increase the SNR. This is done to reduce noise and to overcome problems caused by neuroanatomic variability within the gender and age groups.

\section{Statistical Methods}

A set of statistical analyses were performed using the Civet pipeline, Matlab 7 and SPSS 15.0 conducting general linear models, curve estimation regression, which accounted for the colinearity of the sample. A linear model is applied separately at each vertex $t: Y(t)=\mathbf{X} \hat{I}^{2}(t)+\varepsilon(t)$, where $Y(t)$ is the measure of cortical thickness. $\mathbf{X}$ is the matrix of explanatory variables. $\hat{I}^{2}$ represents the slope to be estimated for each explanatory variables, and $\varepsilon(t)$ is the normally distributed error. A series of statistical tests, such as a $t, F$, or adjusted $R^{2}$ values, can be applied. The regression slope, $\hat{I}^{2}$, can also be plotted at every vertex. The ability to derive meaning out of the regression slope is one of the key strengths of cortical thickness analysis since that slope can be expressed as millimeters change. An optimum thresholding index, which maximizes true-positives against both false-negatives and false-positives, was found to lie at $t=3.3$. The threshold for statistical significance was set at an $\alpha$ of 0.01 . Correction for multiple comparisons was needed to control the false-positive rate. The false discovery rate (FDR) controlling procedure for multiple comparisons is reported to be effective for the analysis of neuroimaging data, which was set at 0.01 .

\section{Results}

(a) Heritability maps: Heritability with a peak value of 0.86 was found for the left cingulated cortex $\left(r^{2}>0.8\right.$, $P<0.01)$; left posterior cingulated cortex $\left(r^{2}>0.8\right.$, $P<0.01)$ and the right anterior cingulated cortex $\left(r^{2}>0.8, P<0.01\right)$. Heritability with a peak value of 0.75 were found in the left medial frontal/motor cortex (BA6) $\left(r^{2}>0.7, P<0.01\right)$. left anterior cingulate cortex $\left(r^{2}>0.6, P<0.01\right)$; and the right insular cortex $\left(r^{2}>0.6, P<0.01\right)$. Such high ICC is suggestive of additive genetic variance (Figs. 1 and 2 ).

(b) Symptoms severity effects on cortical thickness: (1) Regressing cortical thickness with the YGTSS we found significant negative correlations in the right insula with the YGTSS $(r=-0.41, F=6.09$, $P<0.02)$, and the left cingulated cortex with the (CY-BOCS) $(r=-0.35, F=4.30, P<0.05)$.

\section{Discussion}

The main finding of the present preliminary report is the high relatedness/similarity estimates for fraternal siblings concordant for TS $(r=0.86-0.60)$ in the motor-cingulateinsular cortices. Regression analysis revealed significant cortical thinning with increasing tics and obsessive compulsive symptoms in the cingulate and insular cortices.

In TS, the patient who sees an obese person and uncontrollably vocalizes loudly 'fat fat lady' as a verbal tic may have cingulated cortex dysfunction (Devinsky et al. 1995). The authors further stated that: (i) electrical stimulation of the cingulated cortex in monkeys evokes guttural sounds and calls; (ii) connections between the cingulated cortex and structures that are involved in vocalization; (iii) there are similarities between complex, coordinated movement patterns evoked by electrical stimulation of the cingulated cortex in humans and those observed during tics; (iv) a $15 \%$ decrease in cerebral glucose utilization in the cingulate and insular cortices is observed in patients with TS; and (v) lesions of the cingulum bundle can relieve obsessive compulsive symptoms (OCD). Bohlhalter et al. (2006) analyzed functional neuroimaging activities before and at tic onset, of interest to the present study, they identified the cingulate and insular cortices to be predominantly activated. In addition, Kawohl et al. (2008) examined, using functional MRI, three different conditions: "tics", "tics suppressed", and "tics imitated". The comparisons of "tics" to tics suppressed" and of "tics" to "tics imitated" showed similar activation in the cingulated cortex. This finding lends further support to the role of the cingulated cortex in the TS behavioral phenotype. The posterior cingulated cortex has also been involved in TS (Marsh et al. 2007). Furthermore, the significant ICC in the middle frontal/motor cortex, as depicted in Fig. 1, is in accordance with the Leckman et al. (1991) notion that facial tics would be associated with dysfunction in an orofacial subset of the motor circuit, eye blinking with the occulomotor circuit. Based on all of the above, we speculate that the high ICC between siblings and the negative correlation between TS symptoms severity and cortical 
Intraclass coefficient cortical thickness maps in siblings concordant for Tourette syndrome

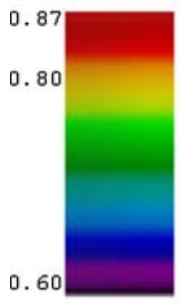

a

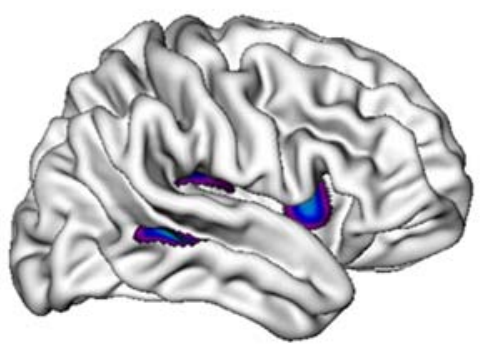

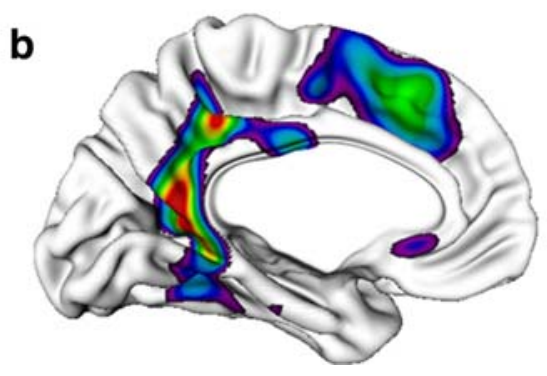

Fig. 1 Intraclass coefficient cortical thickness maps in siblings concordant for Tourette syndrome. a Right lateral sagittal view of the cerebral cortex intraclass coefficient (ICC) map depicting an ICC of 0.60 for the insula. b Right medial sagittal view of the cerebral cortex map depicting significant ICC in the anterior cingulate, posterior cingulate and middle frontal/motor cortices. c Left medial sagittal view of the cerebral cortex depicting an ICC of 0.80 in the

thickness measurements are related to the disturbances in the maturation of the motor-cingulate-insular cortical neural system that mediate self-regulatory processes. Such delayed maturation may consequently contribute to the development of TS by releasing motor and vocal tics from regulatory control. These findings may have important genetic implications. Specifically, Speed et al. (2008) have reported that SLITRK1 is a strong candidate gene for TS. Of particular importance to the present study, SLITRK1 is predominantly expressed in the cerebral cortex (Aruga et al. 2003). Functional and morphological neuroimaging studies investigating the association between cortical thickness measurements and SLITRK1 are needed.

The primary limitation of this study is the lack of a group control. Nevertheless, we have cited several important normal controls studies agreeing that the frontal heritability estimates are quite low in comparison to genetically similar individuals (i.e., siblings and DZ twins who share $50 \%$ of their genes). However, we should note that the sex distribution of the present study is skewed towards malemale sibling pairs. Hence, it may be possible that the observed increases in ICC (relative to these control studies) are related to gender, i.e. that higher correlations are a result of similar "maleness" rather than to TS? Sample size
C

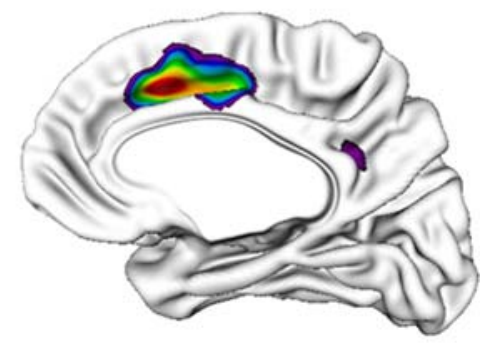

anterior cingulate cortex. The significance of these increased similarities, visualized in color, is related to the local ICC $(r)$. "R-value" refers to the correlation between cortical thickness and genetic liability (siblings) to TS ( $n=16$ pairs). Significantly similar regions are depicted in color. Most of the remaining cortex is significantly less similar between siblings with TS

is another important limitation, which should be acknowledged. Yet it is important to consider the difficulty of recruiting 16 pairs of siblings concordant for TS. In addition, we used a completely automated method to assess cortical thickness. An advantage of an automated method is that rater error is not a factor and corrected for multiple threshold. Third, the common environment shared by the TS fraternal subjects, including early familial factors such as the rearing environment (i.e. shared social and cultural experiences) may have played a role in this high ICC. Of note, we have investigated cerebral cortical thickness, which is mainly constituted of GM. GM is under tight genetic control. Indeed, Plomin and Kosslyn (2001) have stated that high heritability of GM implies that inter-individual variation in cell-body volume is not modified by experience. Common environmental influences would mostly affect the white matter (WM) structures in the brain. Because WM reflects the degree of interconnection between different neurons, interindividual variance in WM volume might be expected to be more under the influence of experience and less under genetic control (Plomin and Kosslyn 2001). Fourth, we have not analyzed the age-byheritability interactions. Age related changes in heritability may be linked to the timing of gene expression and related 
$p$ and standard error maps depicting cingulate-insular thinning as a potential biomarker for Tourette syndrome

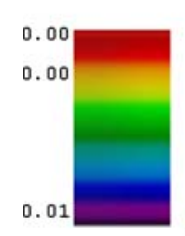

a
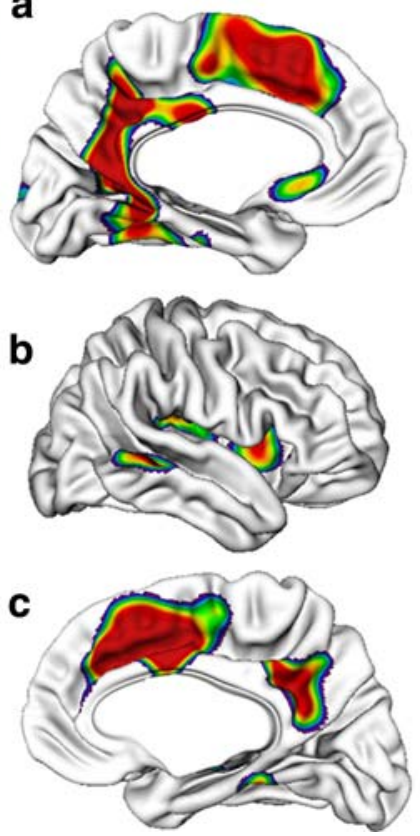

Fig. $2 P$ and standard error maps depicting the motor-cingulateinsular cortices similarities in siblings concordant for Tourette syndrome. The three maps on the left reveal the $P$ value significance of the increased similarities in cortical thickness relatedness/similarity between TS siblings $(n=16)$. The three maps on the right reveal the standard error of the cortical thickness similarities. a Left medial view of the anterior and posterior cingulate cortices and the middle frontal/ motor cortices. b Right lateral view of the insular cortex. c. Right

to the age of onset of disorders. We think that age is important in other neuropsychiatric disorders in which the peak age for the emergence of symptoms is during adolescence. However, the age of onset in TS typically has a prepubertal onset. Fifth, the subjects included in this study were not selected as strictly "pure" TS. They had TS comorbidities, i.e.. attention deficit and hyperactivity disorder (ADHD) and OCD. Of the $34 \mathrm{TS}$ subjects 15 were identified with co-morbid ADHD and 10 with co-morbid OCD. The confounding effect of ADHD in particular has to be considered. The commorbidities between TS and ADHD varies between 21 and 90\% (Robertson and Eapen 1992), and the commorbidities between TS and OCD varies between 40 and 74\% (Robertson 1989), hence came the rational of a confounding effect. Notwithstanding these concerns, the present study is an important approach to help provide a neurobiological marker for TS and may serve as targets for genetic linkage and association studies.

Acknowledgments We thank the subjects who participated in this study. C.F. is a post-doctoral fellow of the Canadian Institutes of Health Research (CIHR), of the CIHR Genes, Mind, Behavior

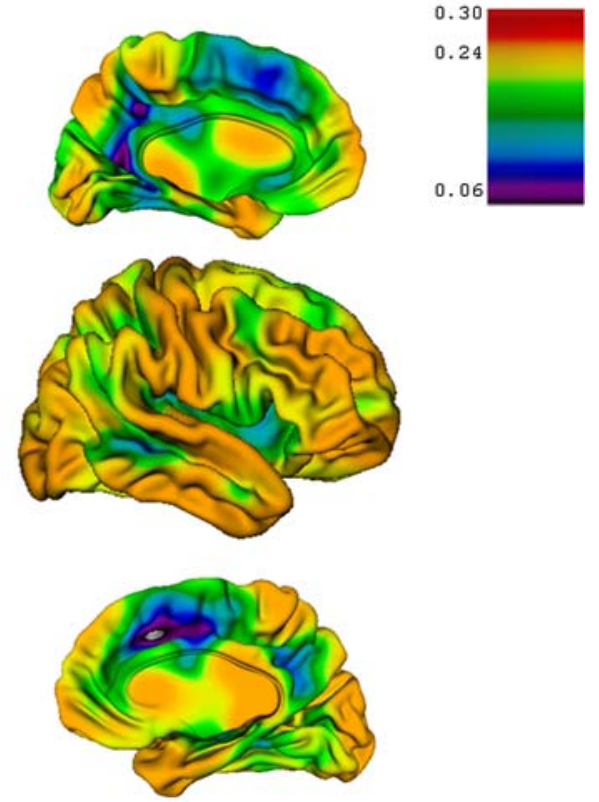

medial view of the anterior and posterior cingulate cortices. Significant similar regions are depicted in red colors for the $P$ values and blue colors for the standard errors. Most of the remaining cortex is significantly less similar between siblings with TS. These results are consistent with the ICC maps presented in Fig. 1 in demonstrating similarities/relatedness in cortical thickness in these regions among TS siblings

Training Program (M. Meany, PI) and the Jeanne Timmins Costello at the Montreal Neurological Institute.

\section{References}

American Psychiatric Association (1994) Diagnostic and statistical manual of mental disorders, 4th edn. American Psychiatric Association, Washington, DC

Aruga J, Yokota N, Mikoshiba K (2003) Human SLITRK family genes: genomic organization and expression profiling in normal brain and brain tumor tissue. Gene 315(2):87-94

Bohlhalter S, Goldfine A, Matteson S, Garraux G, Hanakawa T, Kansaku K, Wurzman R, Hallett M (2006) Neural correlates of tic generation in Tourette syndrome: an event-related functional MRI study. Brain 129(Pt 8):2029-2037

Chung MK, Worsley KJ, Robbins S, Paus T, Taylor J, Giedd JN, Rapoport JL, Evans AC (2003) Deformation-based surface morphometry applied to gray matter deformation. Neuroimage 18(2):198-213

Devinsky O, Morrell MJ, Vogt BA (1995) Contributions of anterior cingulate cortex to behaviour. Brain 118:279-306

Giedd JN, Schmitt JE, Neale MC (2007) Structural brain magnetic resonance imaging of pediatric twins. Hum Brain Mapp 28(6): $474-481$ 
Goetz CG, Tanner CM, Wilson RS, Shannon KM (1987) A rating scale for Gilles de la Tourette's syndrome: description, reliability, and validity data. Neurology 7:1542-1544

Hilgetag CC, Barbas H (2005) Developmental mechanics of the primate cerebral cortex. Anat Embryol (Berl) 210(5-6):411-417

Kawohl W, Bruhl A, Krowatschek G, Ketteler D, Herwig U (2008) Functional magnetic resonance imaging of tics and tic suppression in Gilles de la Tourette syndrome. World J Biol Psychiatry 3:1-4. Retrieved August 27, 2009 from http://www.informa world.com/10.1080/15622970802118356

Laurin N, Wigg KG, Feng Y, Sandor P, Barr CL (2009) Chromosome 5 and Gilles de la Tourette syndrome: linkage in a large pedigree and association study of six candidates in the region. Am J Med Genet B Neuropsychiatr Genet 150B(1):95-103

Leckman JF, Riddle MA, Hardin MT, Ort SI, Swartz KL, Stevenson J, Cohen DJ (1989) The Yale Global Tic severity scale: initial testing of a clinician-rated scale of tic-severity. J Am Acad Child Adolesc Psychiatry 28:566-573

Leckman JF, Knorr AM, Rasmusson AM, Cohen DJ (1991) Basal ganglia research and Tourette's syndrome. Trends Neurosci 14: 94

Leckman JF, Zhang H, Vitale Lahnin F, Lynch K, Bondi C, Kim YS, Peterson BS (1998) Course of tic severity in Tourette's syndrome: the first two decades. Pediatrics 102:14-19

Leckman JF, Cohen DJ, Goetz CG, Jankovic J (2001) Tourette syndrome: pieces of the puzzle. Adv Neurol 85:369-390

Lenroot RK, Schmitt JE, Ordaz SJ, Wallace GL, Neale MC, Lerch JP, Kendler KS, Evans AC, Giedd JN (2009) Differences in genetic and environmental influences on the human cerebral cortex associated with development during childhood and adolescence. Hum Brain Mapp 30(1):163-174

Lerch JP, Evans AC (2005) Cortical thickness analysis examined through power analysis and a population simulation. Neuroimage 24:163-173

Lyttelton O, Boucher M, Robbins S, Evans A (2007) An unbiased iterative group registration template for cortical surface analysis. Neuroimage 34:1535-1544

Marsh R, Zhu H, Wang Z, Skudlarski P, Peterson BS (2007) A developmental fMRI study of self-regulatory control in Tourette's syndrome. Am J Psychiatry 164(6):955-966

Miodonski A (1974) The angioarchitectonics and cytoarchitectonics (impregnation modo Golgi-Cox) structure of the fissural frontal neocortex in dog. Folia Biol (Krakow) 22:237-279

Oldfield RC (1971) The assessment and analysis of handedness: the Edinburgh inventory. Neuropsychologia 9:97-113

Pauls DL (2003) An update on the genetics of Gilles de la Tourette syndrome. J Psychosom Res 55(1):7-12

Peper JS, Schnack HG, Brouwer RM, Van Baal GC, Pjetri E, Székely E, van Leeuwen M, van den Berg SM, Collins DL, Evans AC, Boomsma DI, Kahn RS, Hulshoff Pol HE (2009). Heritability of regional and global brain structure at the onset of puberty: a magnetic resonance imaging study in 9-year-old twin pairs. Hum Brain Mapp 30(7):2184-2196

Plomin R, Kosslyn SM (2001) Genes, brain and cognition. Nat Neurosci 4(12):1253-1258

Rakic P (1995) A small step for the cell, a giant leap for mankind: a hypothesis of neocortical expansion during evolution. Trends Neurosci 18:383-388

Robbins S, Evans AC, Collins DL, Whitesides S (2004) Tuning and comparing spatial normalization methods. Med Image Anal 8(3):311-323

Robertson MM (1989) The Gilles de la Tourette syndrome: the current status. Br J Psychiatry 154:147-169

Robertson MM, Cavanna AE (2007) The disaster was my fault! Neurocase 13(5):446-451

Robertson MM, Eapen V (1992) Pharmacologic controversy of CNS stimulants in Gilles de la Tourette's syndrome. Clin Neuropharmacol 15(5):408-425

Scahill L, Riddle MA, McSwiggin-Hardin M, Ort SI, King RA, Goodman WK, Cicchetti D, Leckman JF (1997) Children's Yale-Brown Obsessive Compulsive Scale: reliability and validity. J Am Acad Child Adolesc Psychiatry 36:844-852

Sled JG, Zijdenbos AP, Evans AC (1998) A nonparametric method for automatic correction of intensity nonuniformity in MRI data. IEEE Trans Med Imaging 17:87-97

Speed WC, O’Roak BJ, Tárnok Z, Barta C, Pakstis AJ, State MW, Kidd KK (2008) Haplotype evolution of SLITRK1, a candidate gene for Gilles de la Tourette syndrome. Am J Med Genet B Neuropsychiatr Genet 147B(4):463-466

The Tourette Syndrome Association International Consortium for Genetics (2007) Genome scan for Tourette disorder in affectedsibling-pair and multigenerational families. Am J Hum Genet 80:265-272

Thompson PM, Cannon TD, Narr KL, van Erp T, Poutanen VP, Huttunen M, Lönnqvist J, Standertskjöld-Nordenstam CG, Kaprio J, Khaledy M, Dail R, Zoumalan CI, Toga AW (2001) Genetic influences on brain structure. Nat Neurosci 4(12):1253-1258

Verkerk AJ, Cath DC, van der Linde HC, Both J, Heutink P, Breedveld G, Aulchenko YS, Oostra BA (2006) Genetic and clinical analysis of a large Dutch Gilles de la Tourette family. Mol Psychiatry 11(10):954-964

Wallace GL, Eric Schmitt J, Lenroot R, Viding E, Ordaz S, Rosenthal MA, Molloy EA, Clasen LS, Kendler KS, Neale MC, Giedd JN (2006) A pediatric twin study of brain morphometry. J Child Psychol Psychiatry 47(10):987-993

Yoon U, Fahim C, Perusse D, Evans A (2008). Genetic analysis of cortical thickness in 8-year-old twins. In: The 14th international conference on funtional mapping of the human brain, Melbourne, Australia. NeuroImage, vol 41, p S55

Zijdenbos AP, Forghani R, Evans AC (2002) Automatic pipeline analysis of 3-D MRI data for clinical trials: application to multiple sclerosis. IEEE Trans Med Imaging 21:1280-1291 